\title{
INVESTIGATION OF THE INFLUENCE OF GLYCERIN ON RHEOLOGICAL CHARACTERISTICS OF MARZIPAN PASTES WITH DRY MINERALIZED WHEY
}

\author{
Mihailo Kravchenko ${ }^{1}$ \\ m.f.kravchenko@gmail.com \\ Larysa Rybchuk ${ }^{1}$ \\ lorchik88@ukr.net \\ Dina Fedorova \\ dina_fedorova@ukr.net \\ Roman Romanenko \\ Department of Engineering and Technical Disciplines ${ }^{2}$ \\ Romanco@ukr.net \\ Vladimir Piddubnyi ${ }^{1}$ \\ profpod@ukr.net \\ Inna Danyliuk \\ Department of Technology and Organization of Restaurant Management ${ }^{4}$ \\ cherep_inna@ukr.net \\ Karina Palamarek \\ Department of Technology and restaurant business ${ }^{4}$ \\ karinkap55@gmail.com \\ Tatiana Marusyak \\ pp.tatjana@ukr.net \\ Tetiana Nezveshchuk-Kohut \\ t.semenivna@gmail.com \\ ${ }^{1}$ Department of technology and the organization of restaurant business \\ ${ }^{2}$ Kyiv National University of Trade and Economics \\ 19 Kyoto str., Kyiv, Ukraine, 02156 \\ ${ }^{3}$ Department of technology and the organization of restaurant business \\ ${ }^{4}$ Chernivtsi Institute of Trade and Economics of Kyiv National University of Trade and Economics \\ 7 Tsentralna str., Chernivtsi, Ukraine, 58000
}

\section{Abstract}

There is studied the influence of glycerin on rheological characteristics of marzipan pastes with dry demineralized whey (DDW) for determining its rational concentration in the composition of decorative semi-products PKV and MFV. PKV - marzipan pastes with DDW, used for covering confectionary products and as an interlayer; MFV - marzipan pastes with DDW, used for making candies and modeling figured products.

According to research results, it has been established, that an increase of a glycerin concentration in the composition of model mixtures of marzipan pastes PKV (20\% of DDW) and MFV (30\% of DDW) results in decreasing deformation characteristics of a reversible type. Irreversible deformation is constant and doesn't depend on glycerin concentration.

According to results of the analysis of main rheological constants by a diapason of glycerin concentrations, there has been established a gradual decrease of indices of a highly elastic and conventionally instant resilience module of model compositions of marzipan pastes PKV (20\% of DDW) and MFV (30\% of DDW). Viscosity indices of a resilient aftereffect and pliability gradually grow with an increase of a glycerin concentration in the composition of model marzipan pastes PKV (20\% of DDW) and MFV (30\% of DDW). 
Research results testify that the use of glycerin in the composition of marzipan pastes PKV (20\% of DDW) and MFV (30\% of DDW) gives a possibility to increase their elasticity and softness, at that keeping high forming properties.

The conducted studies have proved the availability of producing marzipan pastes with DDW and glycerin. There has been substantiated the rational content of glycerin in the composition of marzipan pastes with DDW that allows to provide given (desirable) rheological characteristics. The rational concentration of glycerin in the composition of marzipan pastes PKV (20\% of DDW), used for covering floury and confectionary products and as an interlayer is $5 \%$ of the total mass of recipe components. The rational concentration of glycerin in the composition of marzipan pastes MFV (30\% of DDW), used for making candies, modeling figured products is $5 \%$ of the total mass of recipe components.

An improved technological solution doesn't complicate the general technological process and doesn't need the additional technical equipment. Rheological characteristics of the developed marzipan pastes correspond to directions of the technological destination and allow to decorate floury confectionary products with different levels of technological complication, providing the maximal beauty of ready products.

Keywords: marzipan paste, dry demineralized whey, glycerin, rheological characteristics.

DOI: $10.21303 / 2504-5695.2020 .001120$

\section{Introduction}

Modern decorative semi-products for confectionary products are presented by the wide assortment of diverse creams, pomades, glaze masses, plastic masses of chocolate, marzipan, sugar, painting masses, powders, molten decoration of caramel, chocolate and so on [1-3]. The special place among decorative semi-products is occupied by marzipan $[4,5]$.

Marzipan belongs to high-caloric semi-products, produced of expensive raw materials, because it contains $80 \%$ of almond kernel. Results of previous studies proved the perspective of using dry demineralized whey (DDW) in the recipe composition of marzipan pastes.

The use of DDW essentially improves the biological value of marzipan pastes at the expanse of 1.4 times protein increase and addition with such limiting amino acids as lysine and threonine. In which result consumption of $100 \mathrm{~g}$ of almond paste with DDW concentration $20-30 \%$ provides the human daily need by $45-64 \%$ in lysine, $65-91 \%$ in threonine [6]. DDW also gives a possibility to decrease the energetic value of marzipan pastes by $29-35 \%$ in first turn as a result of changing the qualitative carbohydrate composition, presented by lactose [6]. Lactose is an energy source for a human and also: participates in such important process as calcium metabolism; stimulates processes of nervous regulation. Its sweetness is 5 times lower, comparing with sucrose. Lactose may be consumed by patients with diabetes mellitus, because its glycemic index is 1.5 times less than one of sucrose $[7,8]$.

There is also established the positive influence of DDW on sensor and rheological characteristics of model compositions of marzipan pastes. Results of complex studies have proved a possibility of a partial replacement of import-depending raw materials in the composition of marzipan pastes, so decrease of the prime cost of ready products.

As a result of the study of surface characteristics of marzipan pastes with DDW, there has been established an increase of their adhesive-cohesive properties. Adhesion is an indirect parameter that characterizes a consistence of a food system and influences its consumption properties. Taking into account the research object, an increase of adhesive-cohesive properties not only regulates a consistence of marzipan pastes, but positively influences the technological process of producing decorative semi-products of it. But at the industrial production of marzipan pastes, especially, formation of candies, an adhesion increase has negative results. Adhesion negatively influences the effectiveness of using the equipment, results in an increase of losses of raw materials and energetic resources. The stickiness of marzipan masses is demonstrated at its contact with construction materials during the whole technological process of making a food product. These properties mainly determine the choice of a construction material, working mode of machines or their working bodies. Taking it into account, the expedience of using glycerin in the composition of marzipan pastes with DDW for regulating their adhesive-cohesive interaction has been proved. It has been experimentally established, that introduction of $4 \ldots 5 \%$ of glycerin in the composition 
of marzipan pastes with DDW allows to decrease the strength of adhesion and to approximate it to ones, produced by the traditional technology. But for determining the rational concentration of glycerin in the composition of marzipan pastes with DDW, it is expedient to consider its influence on rheological characteristics.

\section{Materials and methods}

Resilient-elastic and plastic-viscous properties of model compositions of marzipan pastes, based on determining a shift deformation, related to the thickness of a sample, were studied on the Tolstoy flat-parallel elastoplastometer, Fig. 1 [9, 10].

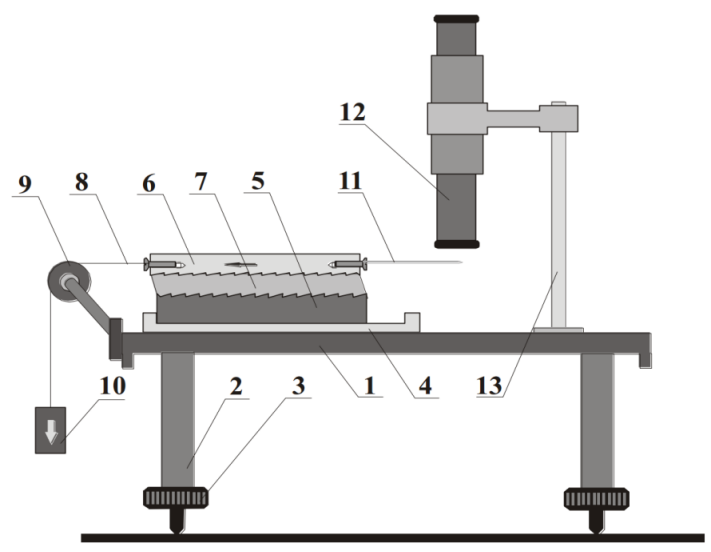

Fig. 1. Setting scheme of the Tolstoy flat-parallel elastoplastometer: 1 - table; 2 - supporting leg; 3 - regulating screw; 4 - metal stand; 5 - metal plate; 6 - plate of plaxiglass; 7 - experimental sample; 8 - silk thread; 9 - block; 10 - load; 11 - observing needle; 12 - microscope; 13 - tripod

The first value of absolute deformation was obtained by the microscope instantly, as soon as the load became to affect the upper plate. After that the value of absolute deformation was fixed with $1 \mathrm{~min}$ periodicity during $10 \mathrm{~min}$. Further observations were conducted with 5 min periodicity. After removing the load, the instant deformation was fixed, then device indications were fixed with $1 \mathrm{~min}$ periodicity during $10 \mathrm{~min}$. At studying rheological characteristics of model compositions of marzipan pastes, the fixed load was selected for all systems as $65 \mathrm{~g}$. At the same time equal temperature $\left(+6^{\circ} \mathrm{C}\right)$ and height of samples $(7 \mathrm{~mm})$ were provided.

For determining rheological parameters, the dependence of relative deformation on time of the tension effect was constructed, Fig. 2. The following segments were separated on it:

$-\gamma_{0}, \varepsilon_{0}$ (segment OA) - resilient conventionally instant deformation that instantly (in a very short time interval) appears under the effect of the applied tension and instantly disappear after removing it; tension;

$-\gamma_{\mathrm{m}}, \varepsilon_{\mathrm{m}}$ (segment OC) - maximally reached deformation under the effect of the applied

$-\gamma_{\text {rev }}, \varepsilon_{\text {rev }}$ (segment OB1) - highly elastic deformation - completely reversible relative deformation;

$-\gamma_{\text {rev }}=\gamma_{0}+\gamma_{\text {rev }}\left(\varepsilon_{\text {rev }}=\varepsilon_{0}+\varepsilon_{\text {he }}\right) ; \varepsilon_{\text {he }}$ (segment AB1) - highly elastic deformation - relative deformation, gradually disappearing after removing the load:

$-\gamma_{\mathrm{he}}=\gamma_{\mathrm{rev}}-\gamma_{0}\left(\varepsilon_{\mathrm{he}}=\varepsilon_{\mathrm{rev}}-\varepsilon_{0}\right)$

$-\gamma_{\text {res }}, \varepsilon_{\text {res }}$ (segment EO1) - residual (plastic) deformation that doesn't disappear after removing the applied tension during infinitely long time: $\gamma_{\text {res }}=\gamma_{\mathrm{pl}}\left(\varepsilon_{\mathrm{res}}=\mathrm{pl}\right)$.

Separation of the total deformation in reversible and irreversible ones is realized by the simplified method of extrapolation of the deformation linear segment to the cross with the Y-axis $\gamma=f(t)$ (offered by Rebinder). It is supposed, that the growing speed of irreversible deformation is stable and doesn't depend on elastic deformation value. If extrapolate the linear segment to null and transfer it to the beginning of coordinates, the growing speed of irreversible deformation is obtained [9]. 


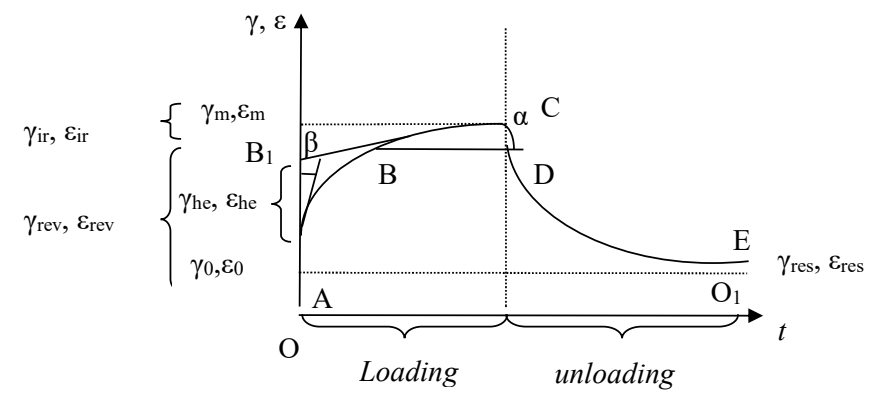

Fig. 2. Creeping curve of a studied sample under the effect of the applied shift tension (at loading and unloading)

The relative deformation is determined by the formula:

$$
\gamma=k x n / d,
$$

where $k$-division value of the microscope, $\mathrm{m} ; n$ - number of divisions on the microscope scale; $d$-sample thickness, $\mathrm{m}$. formula:

The ratio coefficient of reversible deformation to the total one was determined by the

$$
K \gamma=\gamma_{\mathrm{rev}} / \gamma m
$$

where $\gamma_{\text {rev }}$-value of reversible deformation; $\gamma m$ - value of maximal deformation.

The shift tension was determined by the formula:

$$
\tau=m x g / S,
$$

where $\tau$ - shift tension, Pa; $m$ - load mass, $\mathrm{kg} ; g$ - free fall acceleration $\left(9.81 \mathrm{~m} / \mathrm{s}^{2}\right) ; S$ - plate area, $\mathrm{m}^{2}$.

The pliability that characterizes the ability of the studied samples to deformation under the effect of the applied tension is determined by the formula:

$$
I=\gamma m / \tau,
$$

where $I$ - pliability of the system, $\mathrm{Pa}^{-1} ; \gamma m$ - relative maximal deformation.

The module of instant resilience that characterizes the ability of the studied samples to resist proportionally to its deformation was determined by the formula:

$$
G_{\text {resl }}=\tau / \gamma_{0}
$$

where $G_{\text {resl }}$ - module of instant resilience, $\mathrm{Pa} ; \gamma_{0}$ - relative conventionally instant deformation.

The module of elasticity that characterizes the ability to decrease deformation of the studied samples with time after removing the tension was determined by the formula:

$$
G_{\mathrm{el}}=\tau / \gamma_{\mathrm{he}}
$$

where $G_{\mathrm{el}}$ - module of elasticity, $\mathrm{Pa} ; \gamma_{\mathrm{he}}$ - relative highly elastic deformation.

The plastic viscosity that characterizes the ability of the structured condition to flow without destruction under the effect of the constant tension was determined by the formula:

$$
\eta^{*} 0=\tau / \operatorname{tg} \alpha,
$$

where $\eta^{*} 0$ - plastic viscosity, Pa·s; tg $\underline{\ln }$ - tilt angle of the final linear segment of the curve to X-axis. 
The viscosity of the resilient aftereffect was determined by the formula:

$$
\dot{\eta}_{\text {den }}=\tau / \operatorname{tg} \beta
$$

where $\dot{\eta}_{\text {den }}-$ viscosity of the resilient aftereffect, Pa·s; $\operatorname{tg} \beta$ - tilt angle of the initial linear segment of the curve to $\mathrm{X}$-axis.

\section{Results}

For determining the rational concentration of glycerin in the recipe composition of marzipan pastes with DDW, it was added in amount $1 . .6 \%$ of the total mass of dry components (Table 1).

Table 1

The recipe components of model systems of marzipan pastes with DDW, for $100 \mathrm{~g}$

\begin{tabular}{lcccccc}
\hline \multirow{2}{*}{ Raw material name } & \multicolumn{7}{c}{ Samples with adding glycerin, \% } \\
\cline { 2 - 7 } & $\mathbf{1}$ & $\mathbf{2}$ & $\mathbf{3}$ & $\mathbf{4}$ & $\mathbf{5}$ & $\mathbf{6}$ \\
\hline \multicolumn{7}{c}{ DDW 20 \% } \\
\hline Almond kernel & 32.5 & 32.0 & 31.5 & 31.0 & 30.5 & 30.0 \\
Sugar powder & 32.5 & 32.0 & 31.5 & 31.0 & 30.5 & 30.0 \\
Syrup & 14.0 & 14.0 & 14.0 & 14.0 & 14.0 & 14.0 \\
DDW & 20.0 & 20.0 & 20.0 & 20.0 & 20.0 & 20.0 \\
Glycerin & 1.0 & 2.0 & 3.0 & 4.0 & 5.0 & 6.0 \\
\hline & \multicolumn{7}{c}{ DDW 30 \% } & 26.5 & 26.0 & 25.5 & 25.0 \\
\hline Almond kernel & 27.5 & 27.0 & 26.5 & 26.0 & 25.5 & 25.0 \\
Sugar powder & 27.5 & 27.0 & 14.0 & 14.0 & 14.0 & 14.0 \\
Syrup & 14.0 & 14.0 & 30.0 & 30.0 & 30.0 & 30.0
\end{tabular}

The influence of the glycerin concentration on deformation characteristics of model compositions of marzipan pastes with DDW: total, reversible, irreversible, relative deformation was determined (Table 2).

Table 2

The influence of glycerin on deformation characteristics of model compositions of marzipan pastes with DDW $(n=5 ; P \leq 0.05)$

\begin{tabular}{|c|c|c|c|c|c|c|c|c|c|}
\hline \multirow{2}{*}{ Parameter name } & \multirow{2}{*}{\multicolumn{2}{|c|}{ Symbol }} & \multirow{2}{*}{ Control } & \multicolumn{6}{|c|}{ Samples of marzipan pastes with DDW with adding glycerin, $\%$} \\
\hline & & & & 1 & 2 & 3 & 4 & 5 & 6 \\
\hline \multirow{2}{*}{ Reversible deformation, $10^{-3}$} & \multirow{2}{*}{$\gamma_{\mathrm{rev}}$} & PKV & 317.00 & 322.86 & 350.00 & 365.71 & 380.00 & 401.43 & 420.15 \\
\hline & & MFV & 335.71 & 360.00 & 377.14 & 380.00 & 387.71 & 393.71 & 421.15 \\
\hline \multirow{2}{*}{ Irreversible deformation, $10^{-3}$} & \multirow[b]{2}{*}{$\gamma_{\mathrm{ir}}$} & PKV & 12.86 & 12.86 & 12.86 & 12.86 & 12.86 & 12.86 & 12.86 \\
\hline & & MFV & 12.86 & 12.86 & 12.86 & 12.86 & 12.86 & 12.86 & 12.86 \\
\hline \multirow{2}{*}{ Total deformation, $10^{-3}$} & \multirow{2}{*}{$\gamma_{\text {tot }}$} & PKV & 330.00 & 335.71 & 362.86 & 378.57 & 392.86 & 414.29 & 424.22 \\
\hline & & MFV & 348.57 & 372.86 & 390.00 & 392.86 & 408.29 & 414.29 & 424.22 \\
\hline \multirow{2}{*}{ Shift tension, $\mathrm{Pa}$} & \multirow[b]{2}{*}{$\tau$} & PKV & 425.10 & 425.10 & 425.10 & 425.10 & 425.10 & 425.10 & 425.10 \\
\hline & & MFV & 425.10 & 425.10 & 425.10 & 425.10 & 425.10 & 425.10 & 425.10 \\
\hline \multirow{2}{*}{$\begin{array}{l}\text { Ratio of Rel. deformation to } \\
\text { the tot. one }\end{array}$} & \multirow{2}{*}{$\mathrm{K}$} & PKV & 0.96 & 0.96 & 0.96 & 0.97 & 0.97 & 0.97 & 0.97 \\
\hline & & MFV & 0.96 & 0.97 & 0.97 & 0.97 & 0.97 & 0.97 & 0.97 \\
\hline
\end{tabular}


Indices of total deformation grow with the glycerin concentration increase not directly proportionally and depend on the mass share of DDW in the composition of marzipan pastes. So, at DDW concentration $20 \%$ total deformation indices grow depending on glycerin content up to 1.3 times and are 335.71-4224.22, at DDW concentration $30 \%$ grow up to 1.2 times and are $372.86-424.22$ respectively.

The irreversible deformation is constant and doesn't depend on glycerin concentration. The reversible deformation in its turn grows directly proportionally to the total deformation.

According to the results of deformation characteristics of model compositions of marzipan pastes PKV and MFV there were determined main rheological constants by the glycerin concentration diapason (3)-(7).

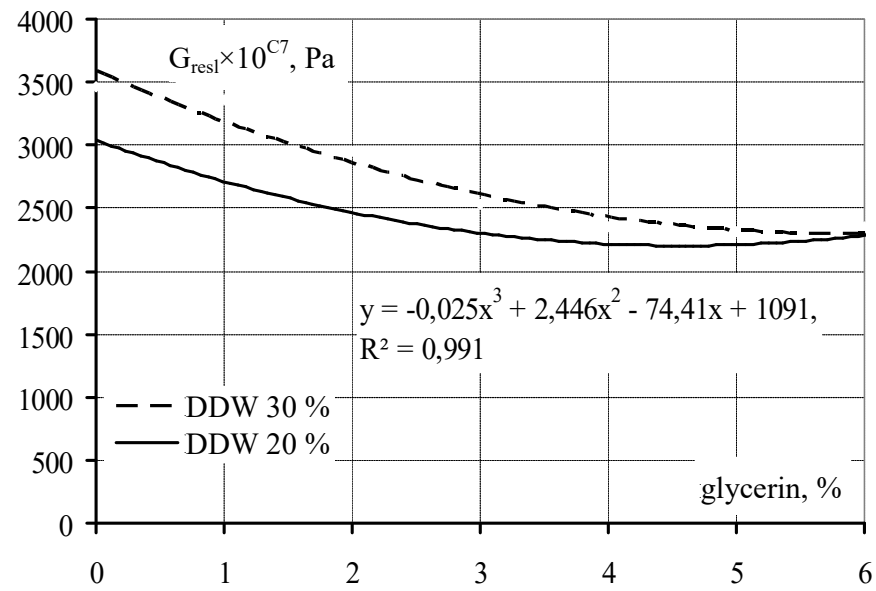

Fig. 3. Influence of glycerin on the conventionally instant resilience module of marzipan pastes PKV (DDW $20 \%$ ) and MFV (DDW $30 \%$ )

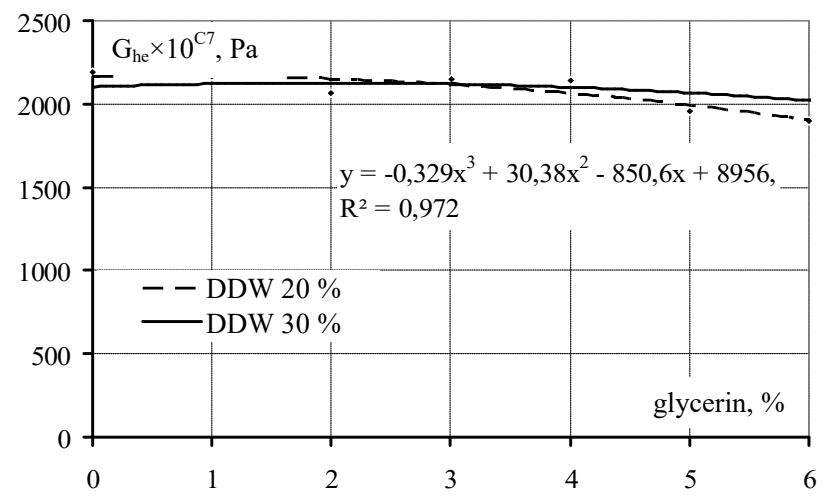

Fig. 4. Influence of glycerin on the highly elastic module of marzipan pastes PKV (DDW $20 \%$ ) and MFV (DDW $30 \%$ )

The dependence of indices of the resilience conventionally constant module (Gresl) of marzipan pastes with DDW on glycerin concentration characterizes the ability of the studied samples to resist proportionally to their deformation (Fig. 3). The indices of the resilience conventionally constant module in the control samples at DDW concentration $20 \%$ are $346.1 \mathrm{~Pa}$, at DDW $30 \%-$ 315.9 Pa. Introduction of glycerin in the recipe composition of marzipan pastes with DDW results in the decrease of the resilience conventionally constant module indices that abruptly lower at glycerin content $2 \%$, then decrease gradually and are $335.5-235.2 \mathrm{~Pa}$ at DDW concentration $20 \%$, 253.3-222.4 $\mathrm{Pa}$ at DDW concentration $30 \%$.

The dependence of indices of the highly elastic module (Gel) of marzipan pastes with DDW on glycerin concentration characterizes the ability to the deformation disappearance in the studied 
samples with time after tension elimination (Fig. 4). The indices of the highly elastic module in the control samples at DDW concentration $20 \%$ are $218.0 \mathrm{~Pa}$, at DDW $30 \%-211.4 \mathrm{~Pa}$. A glycerin concentration has no essential effect on highly elastic module indices, results in their gradual decrease from 216.7 to $211.0 \mathrm{~Pa}$ at DWW concentration $20 \%$, from 221.0 to $200.8 \mathrm{~Pa}$ at DDW concentration $30 \%$ respectively.

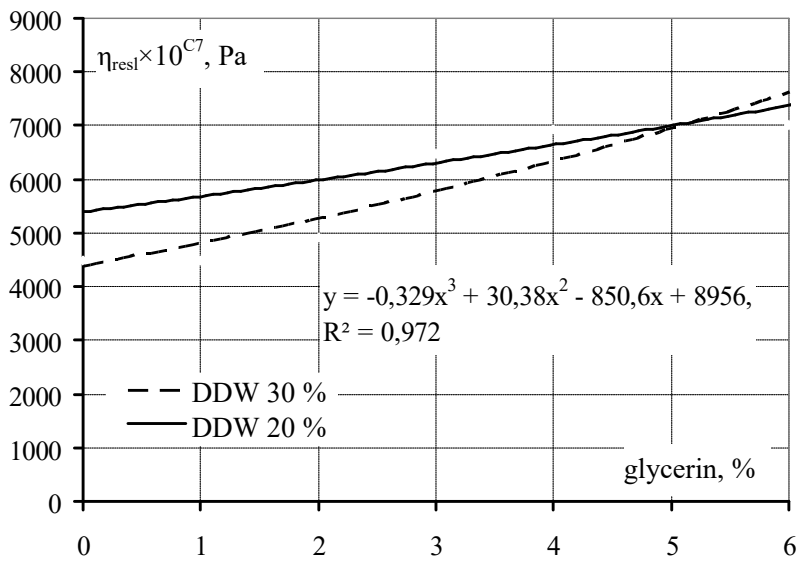

Fig.5. Influence of glycerin on the plastic viscosity of marzipan pastes PKV (DDW $20 \%$ ) and MFV (DDW $30 \%$ )

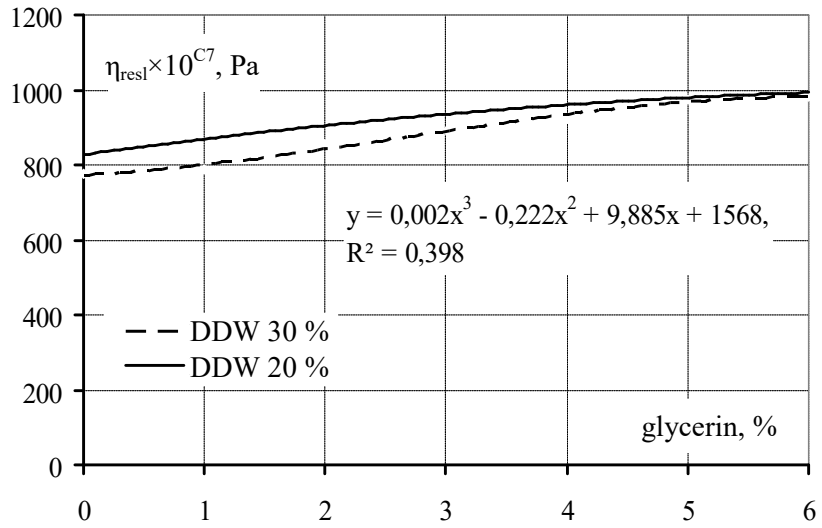

Fig. 6. Influence of glycerin on the resilient aftereffect viscosity of marzipan pastes PKV

(DDW $20 \%$ ) and MFV (DDW $30 \%$ )

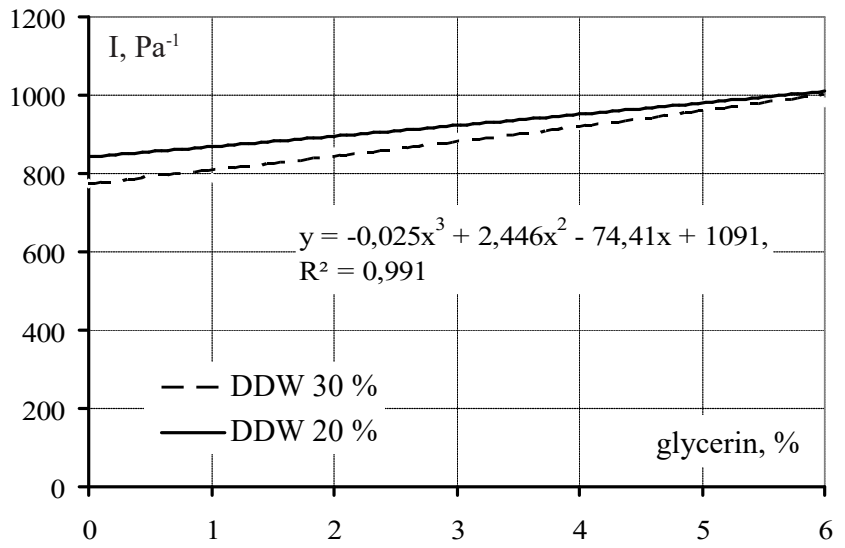

Fig. 7. Influence of glycerin on the pliability of marzipan pastes PKV (DDW $20 \%$ ) and MFV (DDW $30 \%$ ) 
The decrease of indices of the rheological modules with the reversible deformation character favors the plasticity increase. It is testified by the growth of plastic viscosity indices (i) that characterizes the ability of the structured condition to flow without destruction under the effect of constant tension in 1.5 times comparing with the correspondent control samples, Fig. 5. At the expanse of it the formation ability of model compositions of marzipan pastes with DDW improves that is the main criterion of characterizing the consistence of pastes, used for decorating confectionary products.

The glycerin concentration increase results in the growth of viscosity indices of the resilient aftereffect (Fig. 6). At DDW concentration 20\% this index grows up to 1.5 times that is 4485-7001 Pa.s. At DDW concentration $30 \%$ this index grows in 1.2-1.4 times and is 6121$7012 \mathrm{~Pa} \cdot \mathrm{s}$.

Glycerin introduction in the composition of marzipan pastes PKV and MFV results in their softening that makes them more pliable in the process of making decorative semi-products. It is testified by the growth of pliability indices (I) of the food system of marzipan pastes by $40 \%$ (Fig. 7) that characterizes the ability of the studied samples to deformation under the effect of the applied tension.

The growth of viscosity characteristics and pliability of marzipan pastes with DDW is explained by the mobility increase of adsorption layers and weakening of the spatial frame. The further glycerin concentration increase results in destruction of the paste-like structure and loss of the forming ability. So, it is not expedient to increase the glycerin concentration in the composition of marzipan pastes with DDW over $5 \%$.

\section{Conclusions}

The experimental studies of the rheological parameters have established the rational glycerin concentration of marzipan pastes PKV and MFV.

The increase of the glycerin concentration in the composition of model mixtures of marzipan pastes with DDW results in gradually decreasing deformation characteristics of a reversible type. It favors the plasticity increase. As a result the forming ability of marzipan pastes improves. It is testified by the decrease of such rheological constants as conventionally instant module of resilience by $25-30 \%$, highly elastic module by $25-30 \%$, and correspondingly plastic viscosity indices up to $45 \%$.

According to the results of the rheological studies, it is not recommended to increase the glycerin concentration over $5 \%$ of the total mass of dry components of marzipan pastes PKV and MFV, because it results in losing the forming ability.

The obtained research results constructively describe the influence of glycerin on the rheological characteristics of marzipan pastes with DDW that allows to elaborate a technology of new types of them with an increased food and biological value, providing the given rheological characteristics. Depending on technological destination, marzipan pastes have the given consistence parameters, determined by the mass share of used glycerin and DDW. New types of marzipan pastes have rather high quality indices and may be recommended for production in the industry and restaurant economy enterprises for nutrition of different population layers.

But in further it may be expedient to consider the influence of other surface active substances on rheological characteristics of marzipan pastes for a comparative analysis.

\section{References}

[1] Miah, J. H., Griffiths, A., McNeill, R., Halvorson, S., Schenker, U., Espinoza-Orias, N. D. et. al. (2018). Environmental management of confectionery products: Life cycle impacts and improvement strategies. Journal of Cleaner Production, 177, 732751. doi: https://doi.org/10.1016/j.jclepro.2017.12.073

[2] Nazarova, O. U., Chuprina, O. A. (2018). Current status of confectionery production as of a segment of food industry. Ekonomika i suspilstvo, 16, 953-958. Available at: http://economyandsociety.in.ua/journal/16_ukr/145.pdf

[3] Tamova, M. Yu., Shchikarev, A. N., Basyuk, A. S. (2015). Trends and innovations of the global confectionery industry. Nauchnye trudy KubGTU, 14, 176-183. 
[4] Butt, S. S., Mohammed, I. K., Raghavan, V., Osborne, J., Powell, H., Charalambides, M. N. (2018). Quantifying the differences in structure and mechanical response of confectionery products resulting from the baking and extrusion processes. Journal of Food Engineering, 238, 112-121. doi: https://doi.org/10.1016/j.jfoodeng.2018.05.039

[5] Dorn, G., Savenkova, T., Sidorova, O., Golub, O. (2015). Confectionery goods for healthy diet. Foods and Raw Materials, 3 (1), 70-76. doi: https://doi.org/10.12737/11240

[6] Kravchenko, M., Rybchuk, L. (2019). Optimization of the chemical composition of marzipan past. Proceedings of the Tavria State Agrotechnological University, 19 (3), 233-240. doi: https://doi.org/10.31388/2078-0877-19-3-233-240

[7] Hramtsov, A. (2015). Glycoomics clusters of lactose and its derivatives in nanotechnology of living cultures. Foods and Raw Materials, 3 (1), 3-12. doi: https://doi.org/10.12737/11168

[8] Villeneuve, W., Perreault, V., Chevallier, P., Mikhaylin, S., Bazinet, L. (2019). Use of cation-coated filtration membranes for demineralization by electrodialysis. Separation and Purification Technology, 218, 70-80. doi: https://doi.org/10.1016/j.seppur. 2019.02.032

[9] Horalchuk, A. B., Pyvovarov, P. P., Hrynchenko, O. O., Pohozhykh, M. I., Polevych, V. V., Hurskyi, P. V. (2006). Reolohichni metody doslidzhennia syrovyny i kharchovykh produktiv ta avtomatyzatsiya rozrakhunkiv reolohichnykh kharakterystyk. Kharkiv: KhDUKhT, 63.

[10] Shapoval, S. L., Romanenko, R. P., Forostiana, N. P. (2017). Diahnostyka fizychnykh vlastyvostei kharchovykh produktiv. Kyiv: KNTEU, 192. 